\title{
Probing and Controlling Surface Passivation of PbS Quantum Dot Solid for Improved Performance of Infrared Absorbing Solar Cells
}

\author{
Xiaoliang Zhang, ${ }^{1 *}$ Ute B. Cappel, ${ }^{2}$ Donglin Jia, ${ }^{1}$ Qisen Zhou, ${ }^{1}$ Juan Du, ${ }^{1}$ Tamara Sloboda, ${ }^{2}$ \\ Sebastian Svanström, ${ }^{3}$ Fredrik O. L. Johansson, ${ }^{3}$ Andreas Lindblad, ${ }^{3}$ Erika Giangrisostomi, ${ }^{4}$ \\ Ruslan Ovsyannikov, ${ }^{4}$ Jianhua Liu, ${ }^{1}$ Håkan Rensmo, ${ }^{3}$ James M. Gardner, ${ }^{2}$ and Erik M. J. \\ Johansson ${ }^{5 *}$
}

${ }^{1}$ School of Materials Science and Engineering, Beihang University, Beijing 100191, China

${ }^{2}$ Division of Applied Physical Chemistry, Department of Chemistry, KTH Royal Institute of Technology, SE-100 44 Stockholm, Sweden

${ }^{3}$ Division of Molecular and Condensed Matter Physics, Department of Physics and Astronomy, Uppsala University, Box 516, SE-751 20 Uppsala, Sweden

${ }^{4}$ Institute Methods and Instrumentation for Synchrotron Radiation Research, Helmholtz-Zentrum Berlin GmbH, Albert-Einstein-Straße 15, 12489 Berlin, Germany

${ }^{5}$ Department of Chemistry-Ångström, Physical Chemistry, Uppsala University, 75120 Uppsala, Sweden

* E-mail: xiaoliang.zhang@ buaa.edu.cn; erik.johansson@kemi.uu.se 


\section{Content:}

Figure S1. Light absorption and photoluminescence spectra of PbS CQDs in octane.

Figure S2. TEM image of the CQD-OA.

Figure S3. Steady-state PL spectra of CQD-SSLX and CQD-LSLX solid film prepared on the microscopy slide.

Figure S4. Core level spectra.

Figure S5. Cross-sectional SEM image of the SSLX based CQD solar cell.

Figure S6. Statistics results of photovoltaic parameters.

Figure S7. Integrated photocurrent from IPCE spectra.

Figure S8. Light absorption spectra of the CQD-SSLX and CQD-LSLX film.

Figure S9. Stability of the CQD solar cells.

Figure S10. Mott-Schottky curves of the CQD solar cells.

Figure S11. Conductivity measurement of the CQD solid prepared with SSLX and LSLX method.

Table S1. Fitted parameters of the transient photovoltage curve of the CQD solar cells. 


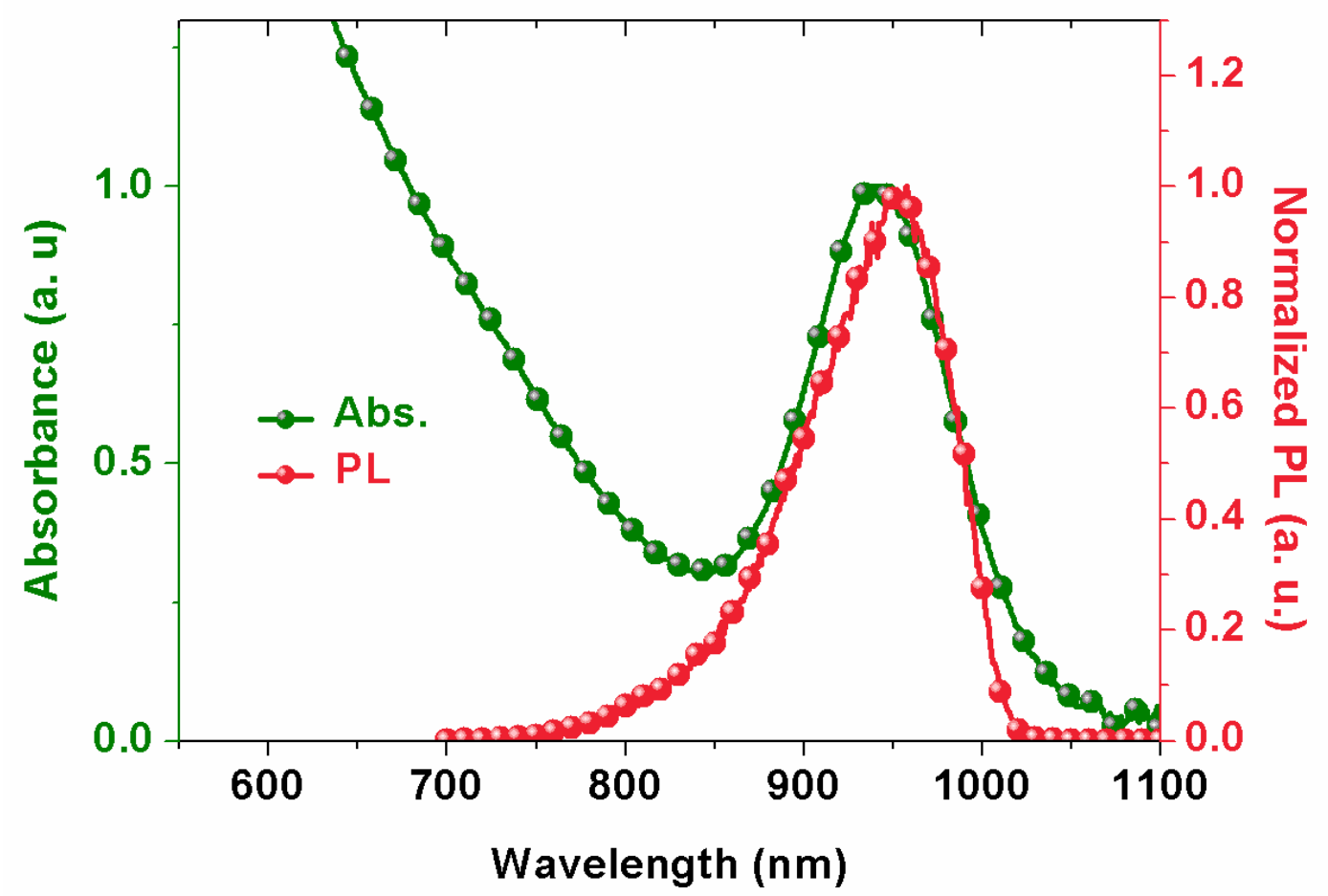

Figure S1. Light absorption and photoluminescence spectra of PbS CQDs in octane. 


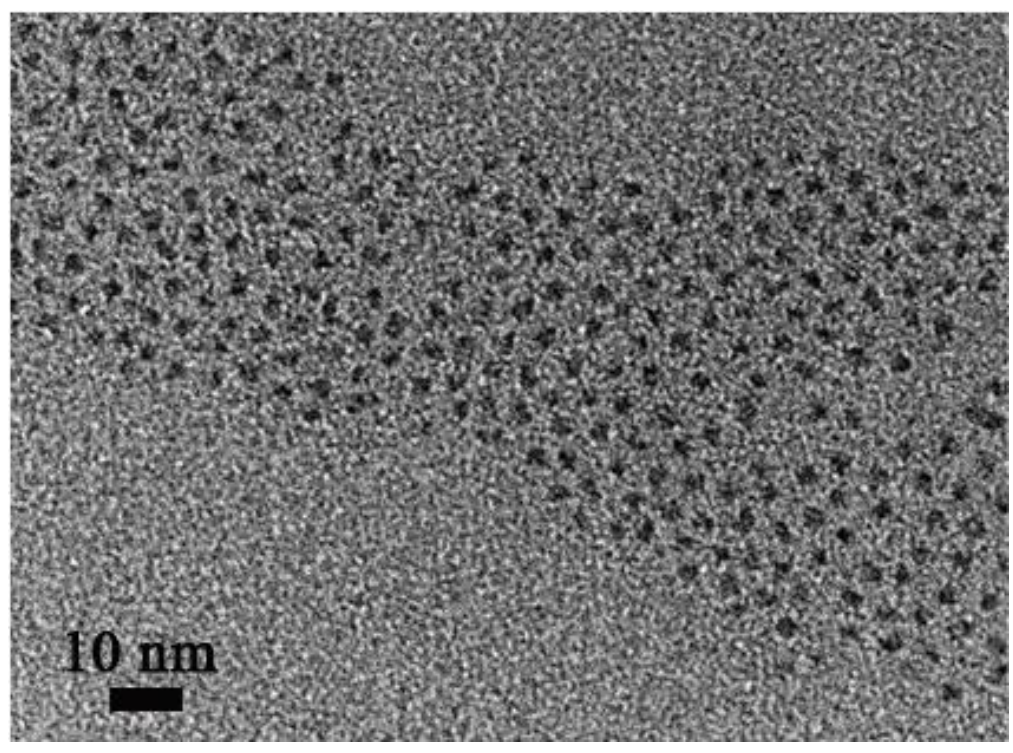

Figure S2. TEM image of the CQD-OA.

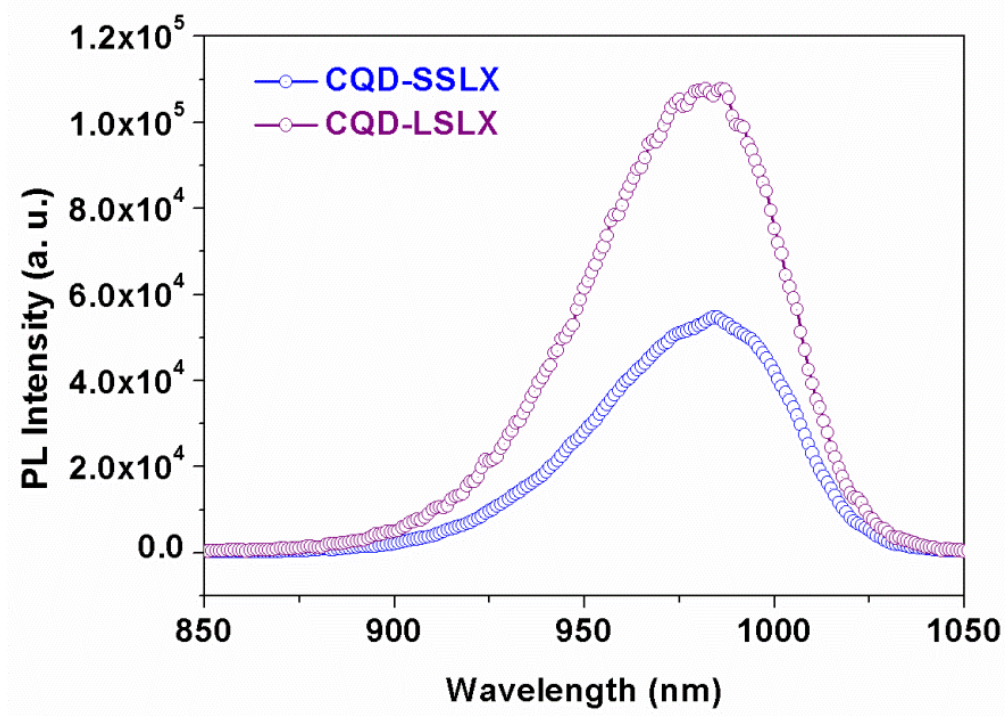

Figure S3. Steady-state PL spectra of CQD-SSLX and CQD-LSLX solid film prepared on the microscopy slide. 

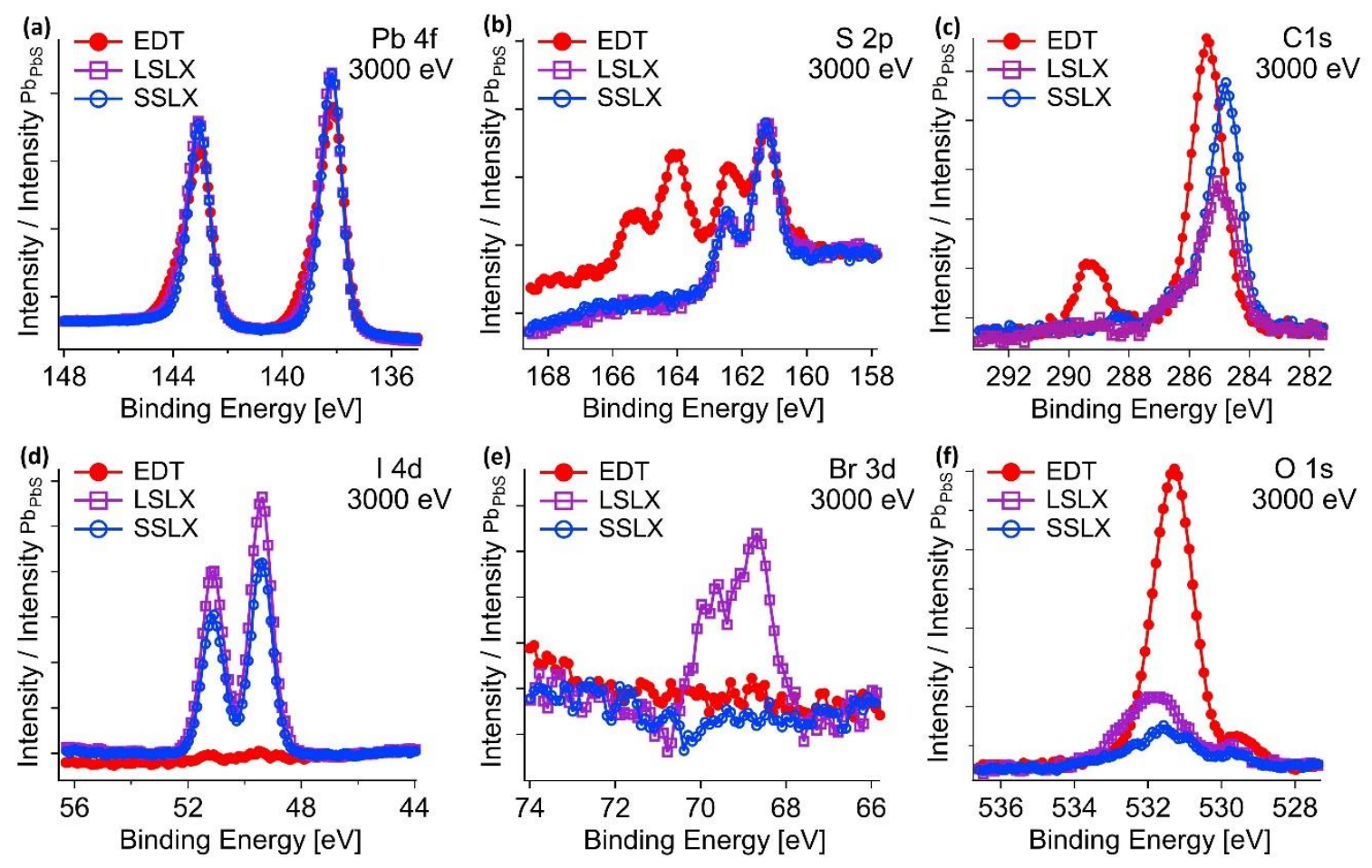

Figure S4. Core level spectra of (a) Pb 4f, (b) S 2p, (c) C 1s, (d) I 4d, (e) Br 3d and (f) O $1 \mathrm{~s}$ of CQD solid obtained at $3000 \mathrm{eV}$ photon energy. Intensities are normalized to the corresponding $\mathrm{Pb}$ intensity obtained from the $\mathrm{Pb} 4 \mathrm{f}$ and $\mathrm{Pb} 5 \mathrm{~d}$ core level fitting $(\mathrm{Pb}$ associated with $\mathrm{PbS}$ ). 


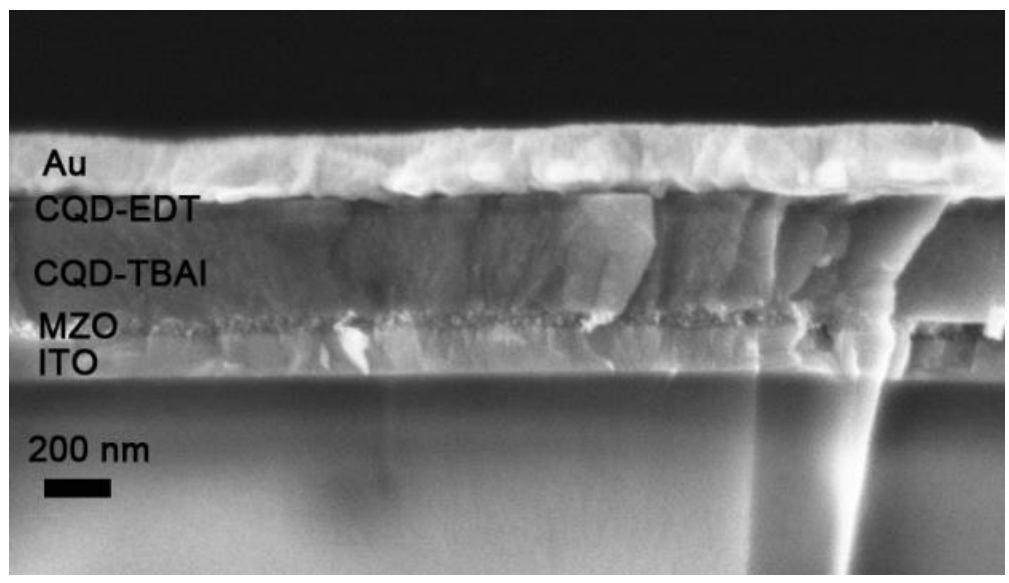

Figure S5. Cross-sectional SEM image of the SSLX based CQD solar cell.
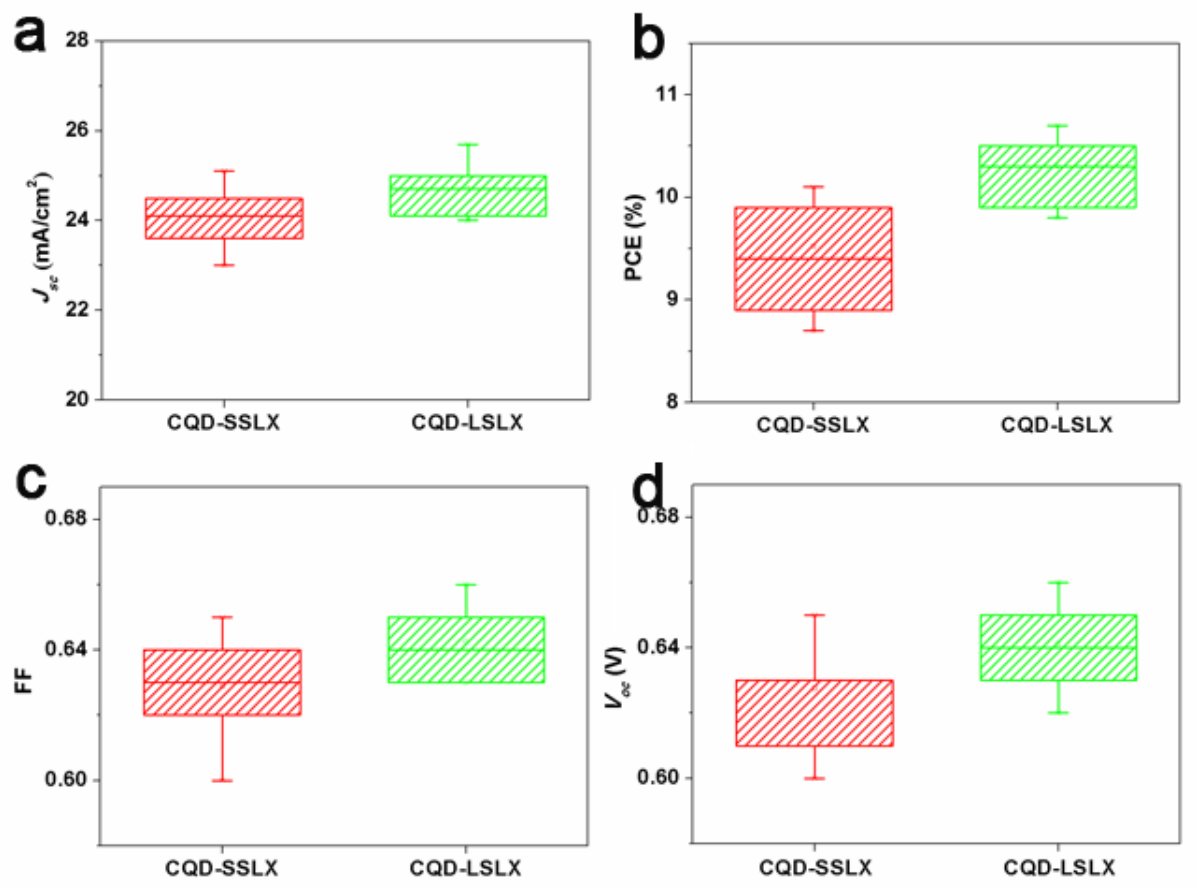

Figure S6. Statistics results of photovoltaic parameters of the CQD-SSLC and CQD-LSLX based solar cells. 16 solar cells from different branches were applied for statistics. 


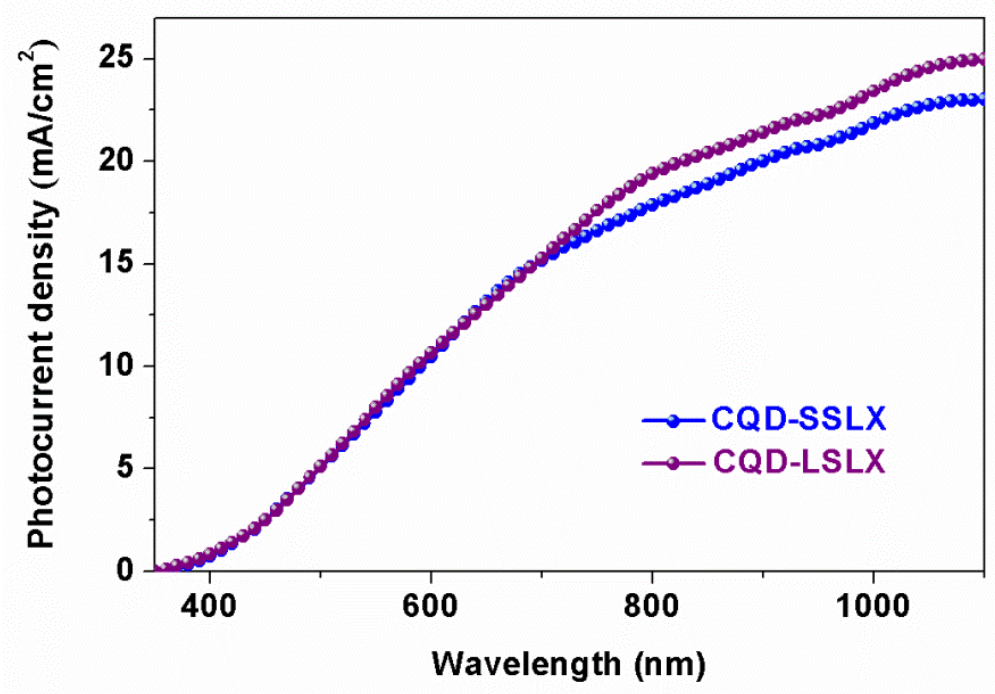

Figure S7. Integrated photocurrent from IPCE spectra.

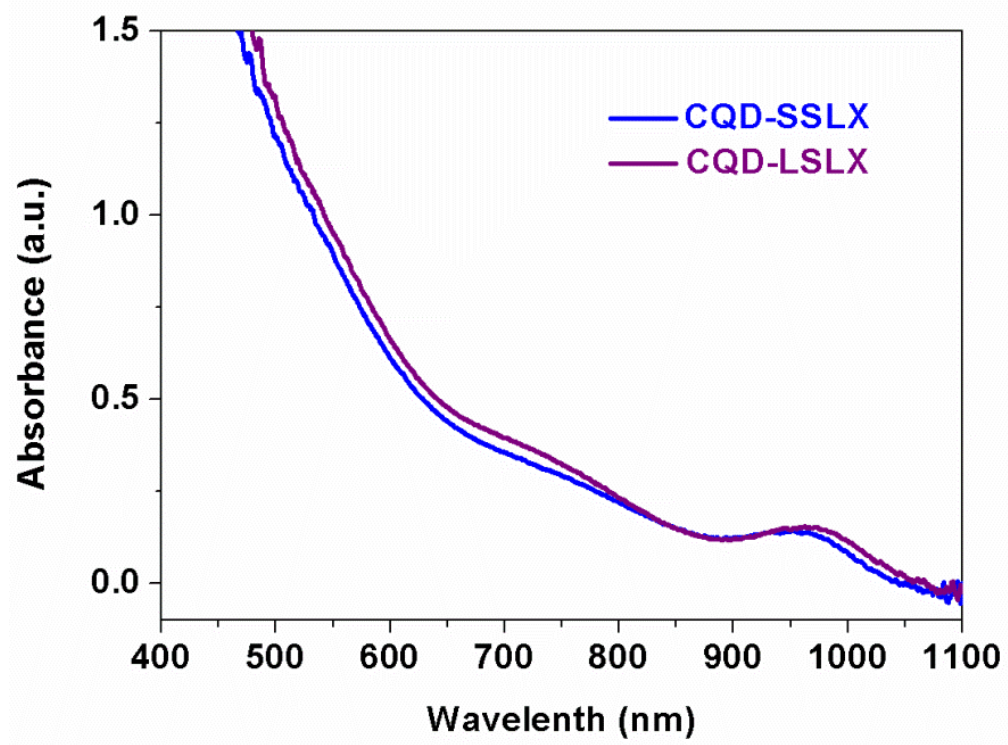

Figure S8. Light absorption spectra of the CQD-SSLX and CQD-LSLX film. 


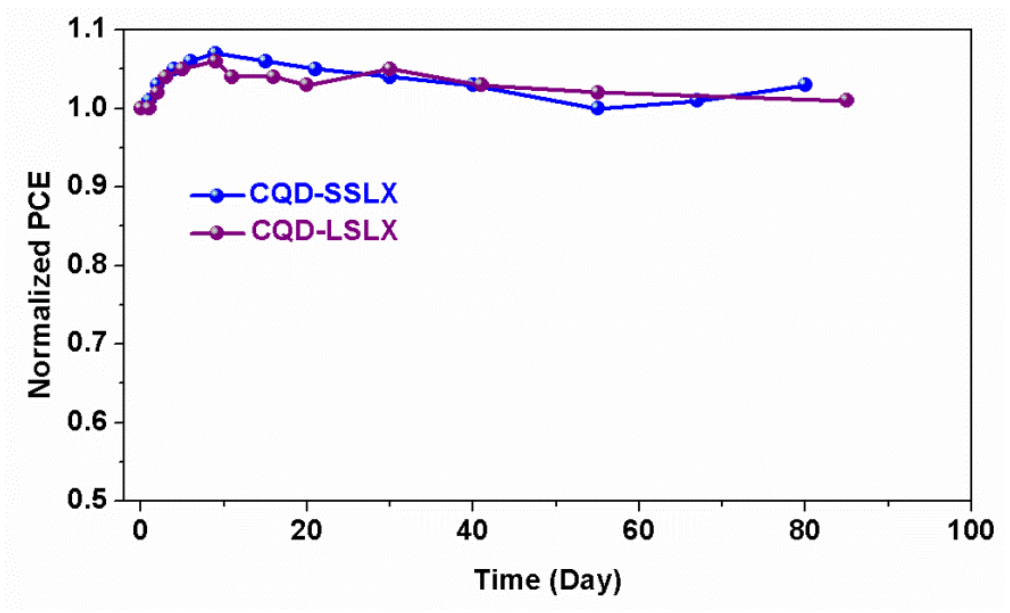

Figure S9. Stability of the CQD solar cells stored under the ambient conditions.

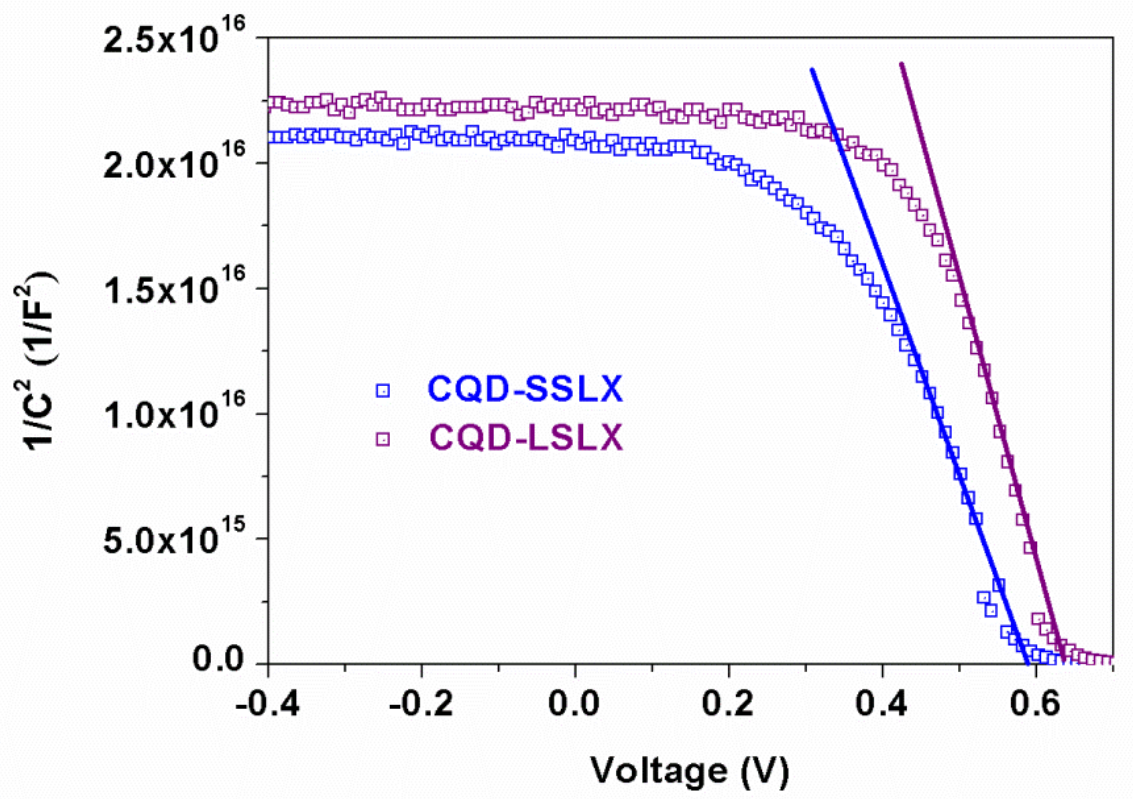

Figure S10. Mott-Schottky curves of the CQD-SSLX and CQD-LSLX based solar cells. 


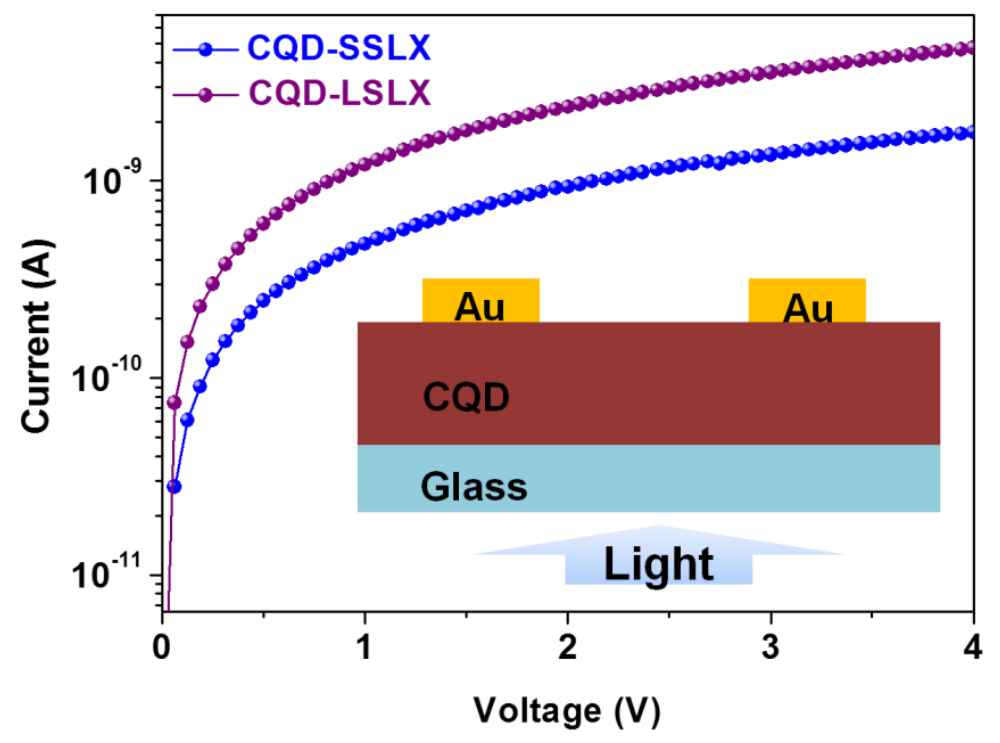

Figure S11. Conductivity measurement of the CQD solid prepared with SSLX and LSLX method.

Table S1. Fitted parameters of the transient photovoltage curve of the CQD-SSLX and CQDLSLX based solar cells.

\begin{tabular}{|c|c|c|c|c|c|}
\hline Equation & \multicolumn{5}{|c|}{$V_{O C}=A 1 \exp \left(-\frac{t}{\tau_{1}}\right)+A 2 \exp \left(-\frac{t}{\tau_{2}}\right)+A 0$} \\
\hline Device & $A 1$ & $\tau_{l}(\mathrm{~ms})$ & $A 2$ & $\tau_{2}(\mathrm{~ms})$ & $A 0$ \\
\hline CQD-SSLX & 0.35 & 0.41 & 0.58 & 34.61 & 0.035 \\
\hline CQD-LSLX & 0.57 & 0.68 & 0.34 & 59.85 & 0.04 \\
\hline
\end{tabular}

